\title{
Evaluation of performance, safety, subject acceptance, and compliance of a disposable autoinjector for subcutaneous injections in healthy volunteers
}

\author{
This article was published in the following Dove Press journal: \\ Patient Preference and Adherence \\ 27 September 2010 \\ Number of times this article has been viewed
}

\author{
Cecile Berteau' \\ Florence Schwarzenbach' \\ Yves Donazzolo² \\ Mathilde Latreille ${ }^{2}$ \\ Julie Berube ${ }^{3}$ \\ Herve Abry' \\ Joël Cotten' \\ Celine Feger' \\ Philippe E Laurent ${ }^{\prime}$ \\ 'BD Medical Pharmaceutical Systems, \\ Le-Pont-de-Claix, ${ }^{2}$ Eurofins Optimed \\ Clinical Research, Gières, France; \\ ${ }^{3}$ Statistics, BD Corporate, Franklin \\ Lakes, NJ, USA
}

Objective: A disposable autoinjector was developed for subcutaneous (SC) self-injection by patients with chronic diseases. To verify its performance and evaluate its acceptance, a clinical study was conducted in healthy volunteers, comparing SC injections performed by subjects using the autoinjector with SC injections performed by nurses using a syringe.

Methods: This was a randomized, single-center, crossover study comparing SC self-injection using an autoinjector with SC nurse-administered injection using a syringe. Two volumes $(0.2 \mathrm{~mL}$ and $1 \mathrm{~mL}$ ) were injected into healthy volunteers. Study objectives included assessment of the accuracy and consistency of the volume injected by the injection systems, and skin reaction and pain associated with the injection. The fluid depot in the SC tissue layer was evaluated by ultrasound. Subject acceptance was evaluated using questionnaires on attitudes and emotions towards the injection technique, and challenged by seeking the subjects' preferred system for a final study injection or future treatment.

Results: A total of 960 injections (480 with autoinjector, 480 with syringe) were performed in 40 subjects. There were no significant differences in mean fluid leakage and injected volumes between the systems. Pain associated with the injection was significantly lower with the autoinjector than with the syringe. Local skin reaction at the injection site was overall satisfactory. Injections were appropriately performed by all subjects. At study end, all 40 subjects preferred the autoinjector for a final study injection and for future treatment.

Conclusion: This study indicated that the autoinjector used by the subject was similar to a syringe used by a nurse in terms of performance and safety in administering the injections, and better in terms of pain, overall acceptance, and preference.

Keywords: subcutaneous injection, autoinjector, self-injection, injection pain, preference, acceptance

\section{Introduction}

Chronic autoimmune inflammatory diseases, such as rheumatoid arthritis (RA), multiple sclerosis (MS), and Crohn's disease, are progressive conditions associated with disability, morbidity, and mortality. ${ }^{1}$ The introduction of injectable disease-modifying drugs a decade ago has had a considerable impact on the progression of such diseases, arresting joint deformities in RA and disease exacerbation in MS for extended periods. ${ }^{2}$ Consequently, patients' adherence to treatment has become a major requisite for achieving optimal treatment efficacy and expected therapeutic outcome. ${ }^{3}$ Factors that influence compliance with injectable treatment in RA remain underinvestigated, while more of these newer
Correspondence: Philippe E Laurent

Medical Affairs, Becton-Dickinson Medical

Pharmaceutical Systems, II Rue A.

Berges, F-3880I Le-Pont-de-Claix Cedex, France

$\mathrm{Tel}+33476683548$

Fax +33476689669

Email philippe_laurent@europe.bd.com 
injectable biologics are becoming available for use. ${ }^{4}$ recent report has suggested that for chronic injectable treatment, selfinjection is associated with better compliance than injection by a family member or by a health care professional. ${ }^{4}$

A self-administered injectable drug should provide patients with a better quality of life due to the increased autonomy and flexibility in adhering to the treatment regimen. Ready-to-use, disposable autoinjectors, prefilled with a fixed drug dose, are currently under clinical development.

This article reports the results of a clinical trial evaluating the performance, safety, and subject acceptability of a newly developed, disposable autoinjector for subcutaneous (SC) injection, which has been specifically designed to improve adherence to an injection schedule. The primary study objective was to evaluate the performance of the prefilled autoinjector used by the subjects relative to standard SC injection by nurses using a prefilled syringe. Secondary objectives were to assess the subjects' pain during the injection, skin reaction, and their compliance with the instructions for use (IFU). Finally, subject preference for self-injection or for injection by a nurse was investigated.

\section{Material and methods \\ Study design}

This study was a randomized, single-center, crossover study comparing self-injection with injection by nurses in healthy volunteers. The study was conducted in France after approval by the Ethics Committee, and in compliance with the latest revision of the Declaration of Helsinki, and with Good Clinical Practice according to European directives and French laws. Each subject came for three separate sessions of eight injections. The order of injections was balanced across all subjects in terms of the system, volume, and injection site, and subjects were randomly assigned to a prespecified order of injection (see Figure 1). An independent observer watched each injection and reported noncompliance with the injection procedure according to a preestablished list of items, including subject errors when handling the autoinjector and performing the injection.

\section{Subjects}

Subject selection and recruitment, and the clinical phase of the study were conducted by a French investigation center (Eurofins Optimed, Grenoble, France). The required number of subjects was 40 . The main eligibility criteria were the following: healthy men and women aged 25-35 years or 45-65 years, free of drugs having an impact on pain perception during the clinical investigations (last drug intake at least six elimination half-lives before injection), without previous experience of self-injection or giving injections, and without current visible skin disease at the targeted injection sites. The group aged 25-35 years was selected to correspond with the age of onset of MS, while subjects in the group aged 45-65 years represented those in the RA population. Subject selection was based on reported clinical history, physical examination, blood tests, absence of ongoing chronic disease, and concomitant treatment.

\section{Injection system and injection procedures}

The standard injection system used by the nurses was a $1 \mathrm{~mL}$ sterile, single-use, prefilled glass syringe (BD Hypak ${ }^{\mathrm{TM}}$; BD, Franklin Lakes, NJ), and the investigational system was the newly developed, prefilled, ready-to-use, singleuse autoinjector used by the subject for self-injection (BD

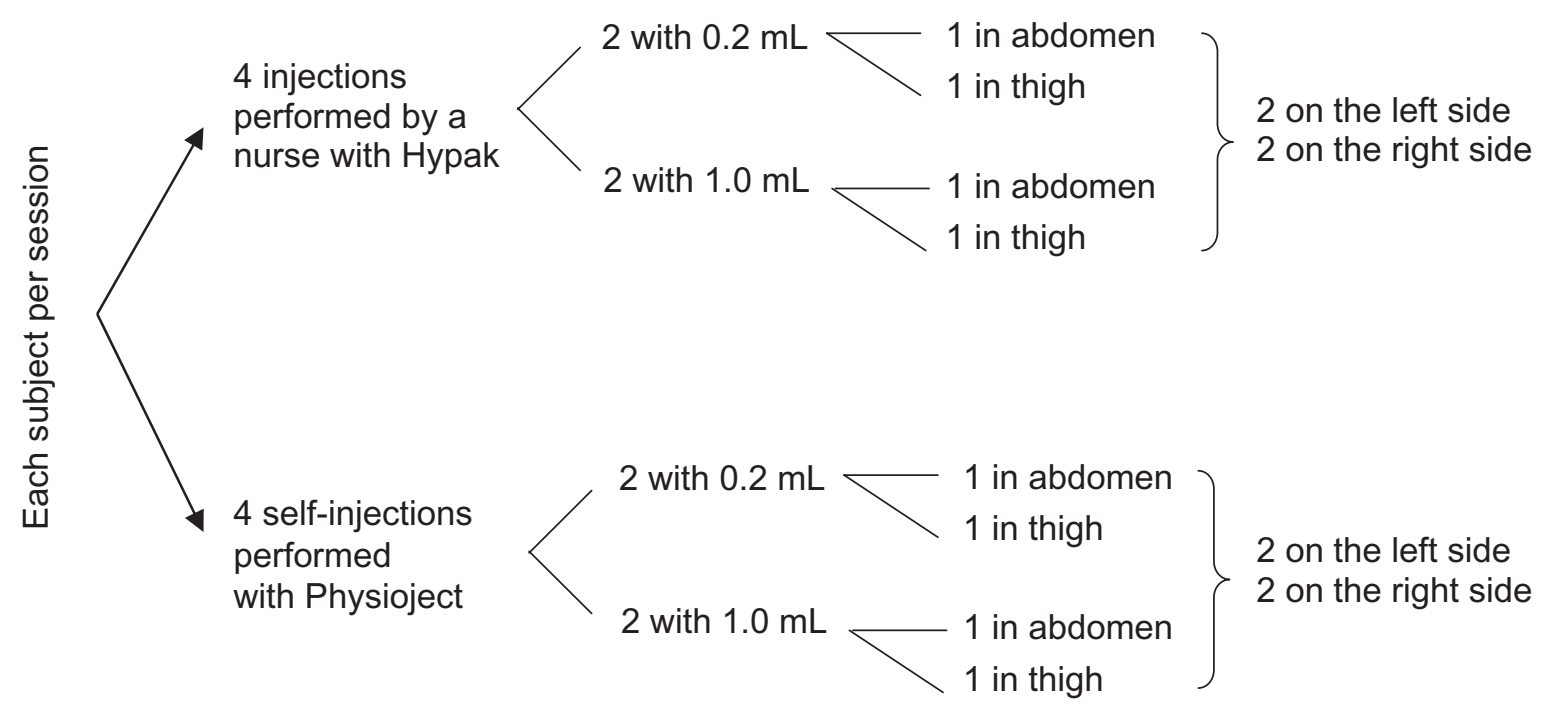

Figure I Study Design - Each subject received a total of 24 injections in three sessions of 8 injections. With 40 subjects, a total of 960 injections were expected. 
Physioject $\left.{ }^{\mathrm{TM}}\right)$. The prefilled BD Hypak syringe and the prefilled syringe assembled inside the autoinjector were the same, with a preattached needle (27 gauge, $12.7 \mathrm{~mm}$ ). Syringes were prefilled with sterile, pyrogen-free, saline $(0.9 \%$ sodium chloride) solution. The two injection volumes evaluated $(0.2 \mathrm{~mL}$ and $1 \mathrm{~mL})$ correspond to the minimum and maximum volumes targeted to be injected with the BD Physioject disposable autoinjector. Prior to the study, all subjects were individually trained to perform SC injections into a skin-fold using the autoinjector. The IFU comprised 10 steps. To counteract any variability between health care professionals on the subjects' cognitive perception of the injection, 14 nurses performed the injections with prefilled syringes, and each subject had a different nurse at each of the three sessions. Nurses performed SC injections according to their usual technique with a skin-fold.

\section{Assessments}

The accuracy and consistency of the injected volume was evaluated by gravimetric methodology using a $0.01 \mathrm{mg}$ precision balance (Sartorius BD211D ${ }^{\mathrm{TM}}$ E2 precision class). ${ }^{5}$ The injection system weight was recorded before and after each injection, and the fluid leakage volume was assessed by collecting the fluid on the skin surface at the injection site immediately after completion of the injection, using ophthalmic sponges. The injected volume was calculated based on the weight of the injection systems before and after injection, and the weight of the sponges before and after leakage collection.

Fluid injection into the hypodermis changes the local ultrasound echostructure of the tissue. Therefore, the injected fluid depot in the hypodermis layer was determined immediately after injection completion using ultrasound sonography (Logiq 500 ${ }^{\mathrm{TM}}, 11 \mathrm{MHz}$ two-dimensional probe, in mode B; GE Medical Systems, Chalfont St Giles, UK). The fluid depot location in the body tissue layers was determined by measuring the distance (in $\mathrm{mm}$ ) between the skin surface and the bottom of the fluid depot. This noninvasive method increased the duration of the subjects' sessions in the clinical center. Because this was an exploratory objective, it was considered sufficient to perform ultrasound assessment on a full set of eight injections from one session only, for all subjects.

Subjects' pain during the injection was measured using a $100 \mathrm{~mm}$ visual analog scale (VAS) ranging from "no pain" to "very painful", and a verbal scale with a choice of eight answers ranging from "I did not perceive anything" to "I perceived an intense pain". ${ }^{6,7}$ double measurement in millimeters (VAS and verbal scale) was performed by two clinical trial technicians. Subjects completed one VAS and one verbal scale immediately after each injection. Local skin reaction was scored after visual inspection of the injection site, recording redness (erythema), bleeding, bruising, edema, and itching. These criteria were used to determine each subject's acceptance of the treatment. ${ }^{7}$

The overall subject preference regarding self-injection compared with injection by a nurse was evaluated using specifically designed questionnaires assessing a subject's emotions and attitudes. ${ }^{8-10}$ Evaluations were made before initiating the investigation and after each session. At the end of the last injection session, subjects were asked to choose between self-injection and injections by nurses for an additional unscheduled injection. In addition, they were asked which combination of systems they would prefer should they ever need a chronic treatment administered by $\mathrm{SC}$ injection, ie, a nurse giving the injection using a syringe or an autoinjector, or performing self-injection themselves using a syringe or an autoinjector.

\section{Data analysis}

Data were analyzed using the $\mathrm{SAS}^{\circledR}$ computer program (version 8.2; SAS Institute, Cary, NC). With regard to the primary objective, a 95\% confidence limit for the 95th percentile of the "fluid leakage" population was calculated per system and per injection volume. The $95 \%$ upper confidence limit was compared with $10 \mu \mathrm{L}$ for the $0.2 \mathrm{~mL}$ injection volume, and with $50 \mu \mathrm{L}$ for the $1 \mathrm{~mL}$ injection volume. A repeated-measures mixed model with subjects as random effects, device, gender, session, and site as fixed effects, and age as a covariable was used to compare performance (average fluid leakage volume and injected volume), average pain, and average fluid depot depth between the two systems per body site and per injection volume. In the presence of non normality, analysis was performed after a normalizing transformation of the data. Skin reactions were analyzed using ordinal logistic regression (edema and bleeding), or Fisher's exact test when the number of positive observations was small (itching, bruising, and erythema). Data on compliance with self-injection IFU were analyzed using a mixed model, whereas data on preference, emotions, and attitudes were analyzed using comparative $t$-tests and, where relevant, analysis of variance.

Skin reactivity and acceptance were analyzed on the safety set corresponding to all injections of all subjects randomized who had at least one injection, including subjects prematurely withdrawn. The per protocol (PP) set corresponded to the safety set with no major protocol deviation. Injection system performance (except for ultrasound assessment) and subject pain were analyzed on the PP set, taking into account all 
injections (24 per subject, 40 subjects, ie, 960 injections). Ultrasound assessment was analyzed on the restricted subset of eight injections per subject in the PP set (ie, 320 injections). Compliance with self-injection IFU was analyzed on the PP set, taking into account all self-injections (ie, 480 injections with the autoinjector). Subject preference was analyzed on the PP set, taking into account the opinion of each subject (ie, 40 subjects' answers).

\section{Results}

\section{Analysis of population}

Of the 62 subjects screened, a total of 40 healthy volunteers were enrolled, as described in Table 1. All 960 planned injections were performed and analyzed. Due to the absence of premature withdrawal, the safety set and PP populations were the same, and had an effective of 960 injections (480 with syringes, 480 with autoinjectors).

\section{Injection system performance}

The data summary results on fluid leakage and injected volume are reported in Table 2. Fluid leakage was not collected for 88 injections, mostly because of blood droplets at the injection site. The mixed-model analysis indicated no significant effect of testing factors on leakage volume and injected volume. The only exception to this was gender, but this effect was not clinically significant, ie, leakage volume was higher in female $(2.7 \mu \mathrm{L})$ than in male subjects $(1.6 \mu \mathrm{L}$, $P=0.0003)$, and injected volume was higher in male $(0.207$ and $1.014 \mathrm{~mL})$ than in female subjects $(0.206$ and $1.011 \mathrm{~mL}$, respectively, $P=0.0037$ ). The rate of injected volume below the expected injected volume was six of 480 injections with the autoinjector (five and one injections for $0.2 \mathrm{~mL}$ and $1 \mathrm{~mL}$, respectively) compared with 17 of 480 with the syringes (eight and nine injections for $0.2 \mathrm{~mL}$ and $1 \mathrm{~mL}$, respectively). Whatever the injected volume, the estimate of the 95th percentile of leakage volume and its 95\% confidence interval (CI) were below the threshold of 5\% of injection volume. These results indicate that the accuracy

Table I Screening and inclusion population

\begin{tabular}{llll}
\hline $\begin{array}{l}\text { Screened } \\
\text { subjects }\end{array}$ & $\begin{array}{l}\text { Included } \\
\text { subjects }\end{array}$ & Age strata \\
\cline { 3 - 4 } & $\mathrm{25-35}$ years & $\mathbf{4 5 - 6 5}$ years \\
\hline $\mathrm{N}=62$ & $\mathrm{~N}=40$ & 12 male & 8 male \\
& & 8 female & 12 female \\
& & Mean age (SD) & Mean age (SD) \\
& & $28.8(2.8)$ years & $54.5(5.8)$ years \\
\hline
\end{tabular}

Notes: Nonincluded subjects, $\mathrm{N}=22$. Eight subjects removed their consent, seven subjects didn't satisfy eligibility criteria, and seven subjects were not included because age strata was already full when the center obtained all their screening results. and consistency of the volume injected by self-injection are not significantly different from injections given by nurses.

In total, 320 ultrasound assessments were performed, comprising 160 after injections by a nurse and 160 after selfinjections. For one injection, delivering a fluid volume of $1 \mathrm{~mL}$ by autoinjector, no fluid depot was detected on ultrasound examination. Fluid depot location in the hypodermis after injection was detected using ultrasound sonography for 159 injections across 160 analyzed injection sites. Figure 2 presents a box plot (arithmetic mean, median, and minimum and maximum values) of depth of the injection fluid depot from the skin surface. The mixed-model analysis indicated no significant difference in depth of the fluid depot between the two groups. No effect of body site or of injected volume was detected.

\section{Subject pain, skin reaction, and acceptance}

Pain measured by a VAS immediately after each of the 960 injections is reported in Figure 3 and Table 3. Subjects reported significantly less pain when injections were selfadministered by the autoinjector than when given by nurses with the syringe $(P<0.0001)$, and with an injection of $0.2 \mathrm{~mL}$ rather than $1.0 \mathrm{~mL}(P=0.0003)$. Female subjects reported less pain than male subjects $(P<0.0001)$. Similar significant differences were also recorded using the verbal scale, except for the gender effect. As detailed below regarding subject acceptance, the reduction in pain was associated with a preference for self-injection.

Skin reactions at the injection site immediately after each of the 960 injections are reported in Table 4. The most frequent local reaction was bleeding, seen mostly as a spot of blood corresponding to needle penetration through the skin. This very minor local bleeding was recorded more frequently with self-injections than with syringes ( $43.1 \%$ versus $10.6 \%$, respectively). Local edema was observed in a few cases and was more frequent with injections by nurses.

A total of 15 adverse events occurred following the 960 injections $(15 / 960,1.6 \%)$, all of which were of mild intensity and resolved spontaneously by the end of the study. The only systemic adverse event was vague malaise after manual injection by a nurse. Most adverse events comprised delayed hematoma in 14/960 cases (1.4\%) at the injection site. The autoinjector was responsible for six cases of delayed hematoma and the syringe was responsible for two cases; identification of the causative injection system was not possible in six of 14 cases due to the delay in onset. 
Table 2 Fluid leakage and injected volume, gravimetric method (primary endpoint) - per protocol population ${ }^{\mathrm{a}}$

\begin{tabular}{|c|c|c|c|c|c|c|}
\hline \multirow[t]{2}{*}{ Volume } & \multirow[t]{2}{*}{ System } & \multirow[t]{2}{*}{$\mathbf{N}$} & \multicolumn{2}{|c|}{ Leakage $(\mu \mathrm{L})$} & \multicolumn{2}{|c|}{ Injected volume $(\mu \mathrm{L})$} \\
\hline & & & $\begin{array}{l}\text { Mean } \\
(\mathrm{SD})\end{array}$ & $\begin{array}{l}\text { Median } \\
(M A D)^{\mathrm{e}}\end{array}$ & $\begin{array}{l}\text { Mean } \\
(\mathrm{SD})\end{array}$ & $\begin{array}{l}\text { Median } \\
(\text { MAD) } \\
\end{array}$ \\
\hline \multirow[t]{4}{*}{$0.2 \mathrm{ml}$} & Self-injection/subject & 205 & 1.7 & 1.0 & 206.8 & 207 \\
\hline & & & $(2.4)$ & $(1.0)$ & $(8.0)$ & (4) \\
\hline & Syringe/nurse & 233 & 2.1 & 1.0 & 206.6 & 208 \\
\hline & & & $(2.6)$ & $(1.0)$ & $(5.8)$ & $(2)$ \\
\hline \multirow[t]{4}{*}{$\mathrm{l} \mathrm{ml}$} & Self-injection/subject & 206 & 3.3 & 1.0 & 1013.0 & 1015 \\
\hline & & & $(17.5)^{c}$ & $(1.0)$ & $(19.9)$ & $(4)$ \\
\hline & Syringe/nurse & 227 & 1.6 & 1.0 & 1012.0 & 1013 \\
\hline & & & $(2.0)$ & $(1.0)$ & $(7.1)$ & (3) \\
\hline \multicolumn{2}{|c|}{ Total of analyzed injections } & \multicolumn{5}{|l|}{871} \\
\hline \multirow{2}{*}{\multicolumn{2}{|c|}{ Missing data }} & \multicolumn{5}{|c|}{ I missing value for an injection by a nurse $\mathrm{b}^{\mathrm{b}}$} \\
\hline & & \multicolumn{5}{|c|}{88 leakages were not collected ${ }^{d}$} \\
\hline \multicolumn{2}{|c|}{ Total of performed injections } & \multicolumn{3}{|l|}{960} & & \\
\hline
\end{tabular}

\section{Compliance with self-injection instructions for use}

Based on the observation by a nurse of the 480 injections performed by the subjects with the autoinjector, the mean percentage of steps performed in compliance with IFU was 92.9\% (95\% CI: 92.3\%-93.6\%). An age effect was detected, with greater compliance among younger subjects $(93.6 \%$ in the group aged $25-35$ years versus $92.0 \%$ in the group aged $45-65$ years, $P=0.0163$ ), but this is not clinically relevant.

\section{Subject preference for self-injection, emotions, and attitudes}

The 40 subjects completed a questionnaire about their emotions, attitudes, and preference. Subjects' emotions regarding self-injection were reflected in words such as "pleasant",

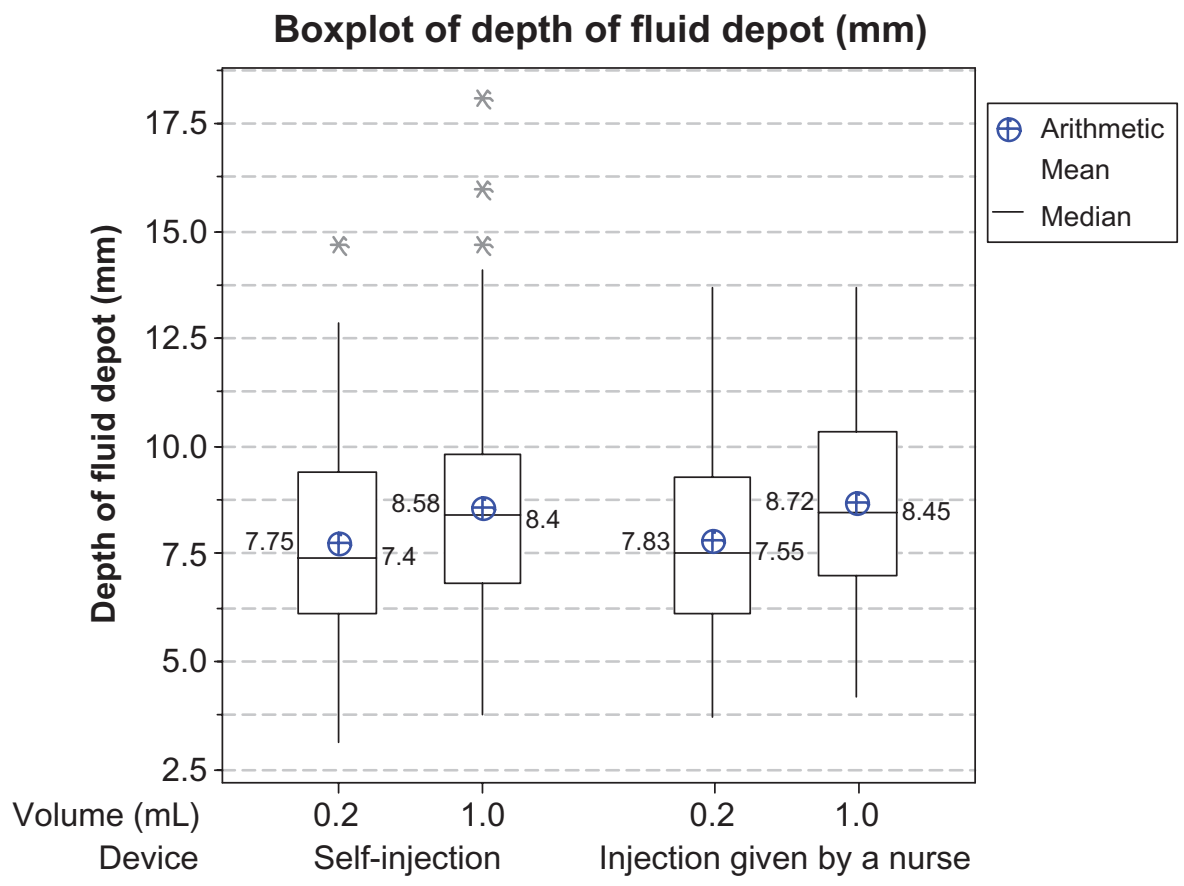

Figure 2 Box plots of depth of fluid depot in $\mathrm{mm}$ from skin surface after injection (ultrasound examination data). Description by device and volume for the per protocol population (mean, median, 25th and 75th percentiles, and range).

Note: *Statistically outlying values. 
$0.2 \mathrm{~mL}$

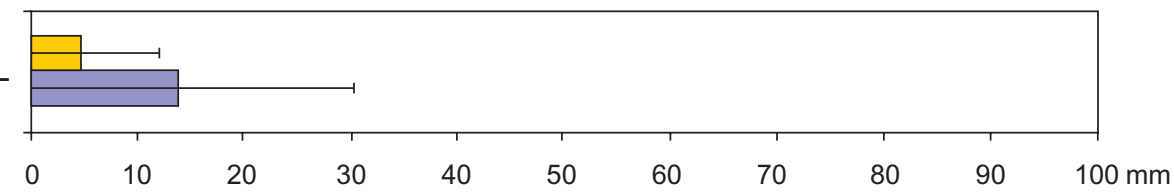

$1.0 \mathrm{~mL}$

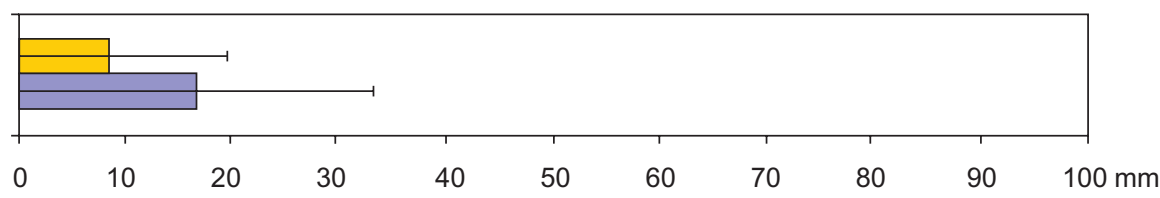

Self-injection/Subject

Syringe/Nurse

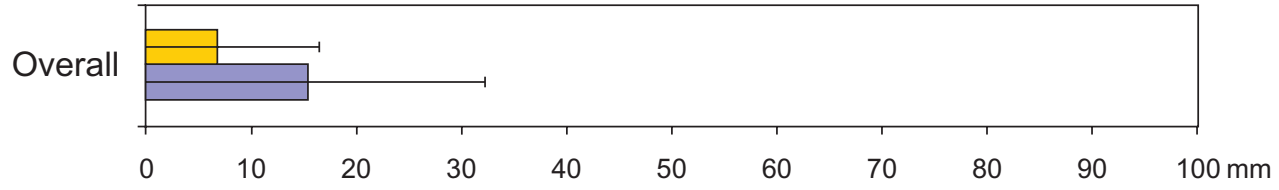

Figure 3 Perceived pain, 100 mm visual analog scale (VAS; secondary endpoint) - per protocol population.

"comforting", "reassuring", and "less anxious" (14/40, 35\%). Ease of use $(10 / 40,25 \%)$ and the autonomy associated with self-injection (8/40, 20\%) were also deemed important.

A majority of subjects reported positive attitudes and emotions with self-injection. However, statistical analysis showed that injection by a nurse was perceived as "easier", "reliable", and more "reassuring" compared with self-injection.

When subjects were asked which injection system they would prefer for an additional unforeseen injection, all subjects selected self-injection. In the event that they ever needed chronic treatment administered by SC injection, subjects ranked self-injection with the autoinjector first (Figure 4).

\section{Discussion}

This study is the first clinical trial in humans evaluating the newly developed autoinjector BD Physioject. Because the main objectives were to document the intrinsic performance, safety, and subject acceptance of the injection system, the trial was conducted in healthy volunteers using a placebo solution. The demographic characteristics of the enrolled subjects were well matched to those reported for MS and RA populations. ${ }^{11,12}$

Ultrasound is an accurate method of measuring depth of the fluid depot in SC tissue. It has been used in previous studies at similar injection sites, and in subjects of similar age and gender to those participating in this study. We used a twodimensional ultrasound method with an $11 \mathrm{MHz}$ probe. The reliability of this imaging technology was confirmed by two previous studies using two-dimensional ultrasound assessment to detect, respectively, $0.1 \mathrm{~mL}$ and $0.5 \mathrm{~mL}$ injections of saline solution in $\mathrm{SC}$ tissue, with a $5 \mathrm{MHz}$ and a $16 \mathrm{MHz}$ probe, respectively. ${ }^{13,14}$

Performance and variability in injected volume accuracy with self-injections were not significantly different from those associated with injections given by nurses. Indeed, regardless of the system, fluid leakages were in the range of a few microliters for a total injected volume of $0.2 \mathrm{~mL}$ or $1 \mathrm{~mL}$. Of note, the volume per injection of most of the drugs approved or in clinical development for autoimmune inflammatory diseases is at least $0.5 \mathrm{~mL}$. In the present study, the rate of injected volume below the expected injected volume was six of 480 injections with the autoinjector compared with 17 of 480 with the syringes.

The injected fluid depot in the hypodermis was observed in $98.8 \%$ of SC self-injections and $100 \%$ of injections administered by nurses. Subjects were asked to make a skinfold, which was not obvious to healthy subjects who are naïve to injection practice, and resulted in skin-folds that were highly variable in size and quality. Skin-folds made by nurses were more alike. One injection fluid depot by self-injection was detected at a depth of $6.6 \mathrm{~mm}$ from the skin surface, most likely because the subject had a very low body mass index $\left(18.3 \mathrm{~kg} / \mathrm{m}^{2}\right)$. Another injection fluid depot was at a depth of $3.3 \mathrm{~mm}$, and categorized as located in the shallow hypodermis. Studies evaluating SC skin thickness using ultrasound evaluation have reported a mean abdomen SC skin thickness ranging from $12.2 \mathrm{~mm}$ to $40.2 \mathrm{~mm}$ according to gender and body mass index, ${ }^{14,15}$ and a mean anterior thigh SC skin thickness ranging from $7.6 \mathrm{~mm}$ to $14.3 \mathrm{~mm} .{ }^{14,16}$ Therefore, a $12.7 \mathrm{~mm}$ depth of needle insertion with a skin-fold procedure 


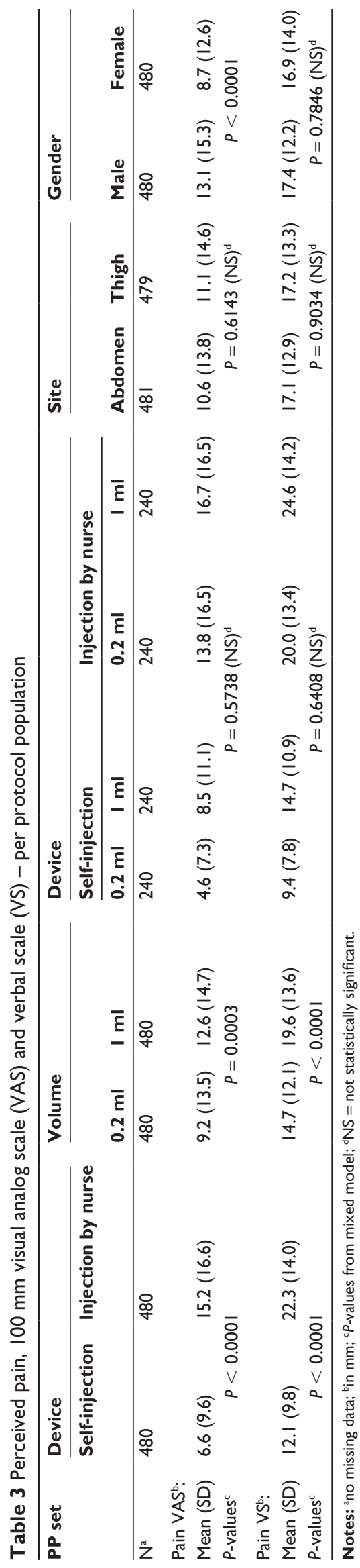

Table 4 Local skin reactions at injection site - Safety population ${ }^{a}$

\begin{tabular}{|c|c|c|c|}
\hline & $\begin{array}{l}\text { Auto-injector/ } \\
\text { subject }\end{array}$ & $\begin{array}{l}\text { Syringe/ } \\
\text { nurse }\end{array}$ & \\
\hline $\begin{array}{l}\text { Bleeding } \\
\mathrm{N}(\%)\end{array}$ & $207(43 . I)$ & $51(10.6)$ & $P<0.000 I^{c}$ \\
\hline $\begin{array}{l}\text { Edema } \\
N(\%)\end{array}$ & $16(3.3)$ & $92(19.2)$ & $P<0.000 I^{c}$ \\
\hline $\begin{array}{l}\text { Bruising } \\
N(\%)\end{array}$ & $3(0.6)$ & I (0.2) & $\begin{array}{l}\text { Not } \\
\text { significant }^{d}\end{array}$ \\
\hline $\begin{array}{l}\text { Itching } \\
N(\%)\end{array}$ & I (0.2) & $0(0.0)$ & $\begin{array}{l}\text { Not } \\
\text { significant }^{d}\end{array}$ \\
\hline $\begin{array}{l}\text { Erythema } \\
N(\%)\end{array}$ & $6(1.3)$ & $5(I . I)$ & $\begin{array}{l}\text { Not } \\
\text { significant }^{d}\end{array}$ \\
\hline
\end{tabular}

Notes: ${ }^{a}$ the safety population includes all subjects with at least one injection; b/32/207 (63.8\%) for Auto-injector/Subject and 30/5I (58.8\%) for Syringe/Nurse are categorized as "just a spot"; ffrom ordinal logistic regression; ‘Fisher's exact test.

should ensure that the injection of fluid is made into the SC tissue layer, regardless of the subject's body characteristics (including body mass index).

Subject pain with the self-injection system was lower than the values usually reported in studies using a $100 \mathrm{~mm}$ VAS, while pain following injection by a nurse was within the previously reported range. ${ }^{7}$ Because pain is recognized as a factor impacting on subject compliance and injection anxiety, many clinical evaluations have investigated possible factors impacting on perceived pain. Needle sharpness and smaller needle diameter contribute significantly to reducing injection pain. ${ }^{7}$ The autoinjector's automatic needle insertion into the skin might therefore contribute to minimizing perceived pain. ${ }^{17}$ Considering the published data and the pain scores reported in the present study, pain was minimized by use of the autoinjector, which combines a prefilled syringe with a $12.7 \mathrm{~mm}, 27$-gauge staked needle and automated needle insertion (8 $\mathrm{mm}$ exposed). Minimal pain was also reflected in the results of the verbal scale, which ranged from "hardly perceptible, without pain" to "perceptible, slightly painful".

Among other factors, slow speed of injection ${ }^{18}$ and the injectable drug formulation (especially fluids that are particularly acidic or basic) $)^{19}$ are reported as significantly influencing perceived pain. In a recent study in 40 healthy volunteers, pain associated with SC injection of a $0.9 \%$ saline solution was scored $14 \pm 20 \mathrm{~mm}$ on a $100 \mathrm{~mm} \mathrm{VAS.}{ }^{20}$ Half of the subjects experienced no pain on injection according to a six-item verbal scale. In another study in 84 healthy volunteers, the pain associated with SC injection of a saline solution was of similar magnitude (mean pain at injection $16.0 \mathrm{~mm}, 95 \% \mathrm{CI}: 12.4 \%-19.7 \%) .{ }^{21} \mathrm{An}$ improvement in drug formulation may also contribute to minimizing perceived pain, and this has been demonstrated 


\section{Interval plot of acceptability \\ $95 \% \mathrm{Cl}$ for the mean}

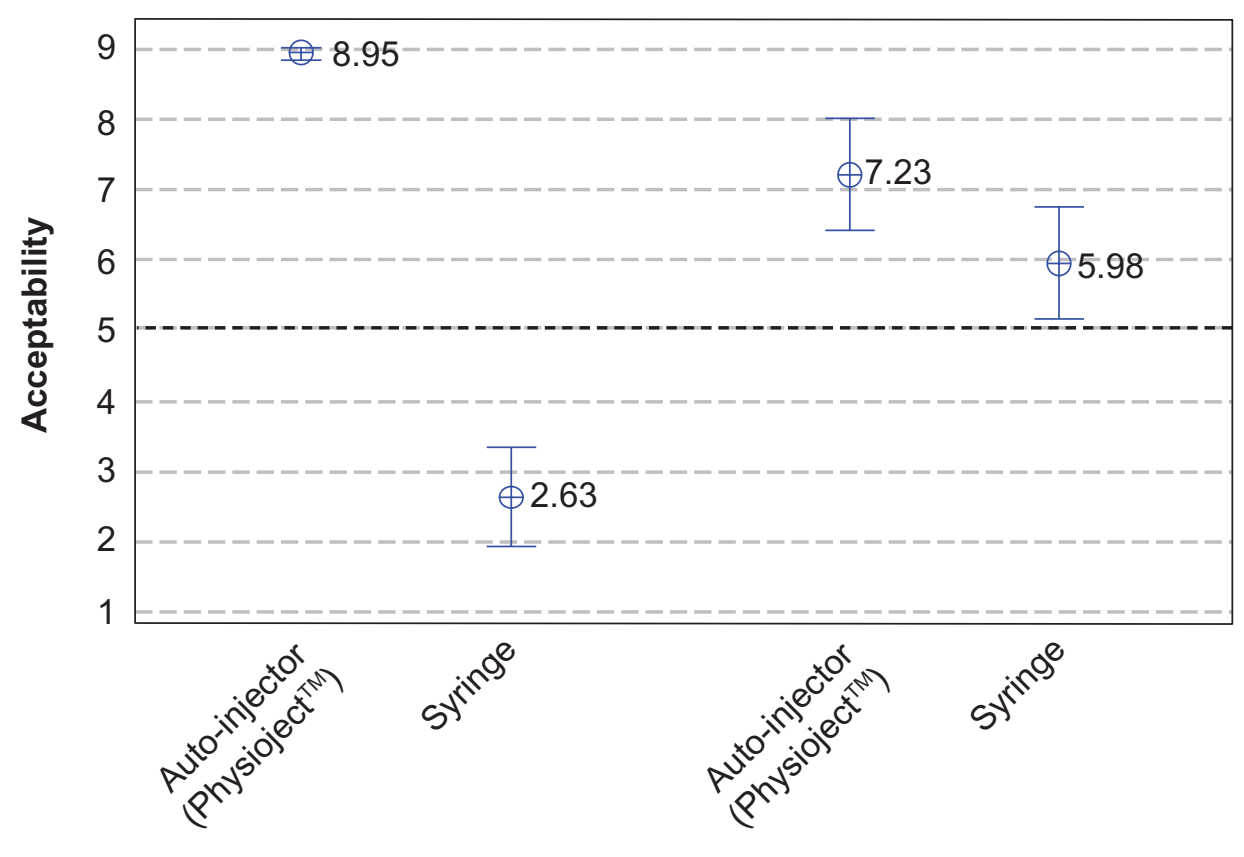

Self-injection

Injection given by a nurse

Figure 4 Device acceptability score for four injection scenarios (mean and $95 \%$ confidence interval).

Notes: At study end the subjects had to answer these four questions with a quotation from I ("Not at all probable") to 9 ("Completely probable"): "For a further chronic treatment administered by SC injection, I agree that I will perform myself these injections with an auto-injector?", I agree that I will perform myself these injections with a syringe?, I agree that a nurse will give me these injections with an auto-injector?, I agree that a nurse will give me these injections with a syringe?

with the improved formulation of interferon beta $1 \mathrm{~b}\left(\right.$ Rebif $^{\mathbb{B}}$; Merck Serono SA, Geneva, Switzerland). It would therefore be of interest to evaluate pain at the injection site using the autoinjector in combination with the drug and in target patient populations.

Local skin reaction may also have a significant impact on patient compliance with chronic treatment with injectables. Local bleeding was more frequent with self-injections than with injections administered by nurses. However, in $63.8 \%$ of injections, bleeding was limited to "just a visible spot" at the injection site and, because the subjects did not spontaneously complain, it is unlikely that this would affect a patient's long-term acceptance of self-injections. Further investigation is warranted in clinical studies with injectable drugs, because bleeding might be influenced by the nature of the injected solution. All other items contributing to local skin reaction were comparable between the autoinjector and the syringe.

Hematomas were reported after injection in a few cases $(15 / 960,1.5 \%)$. The incidence of hematoma is infrequently reported in clinical studies, but the hematoma rate in the present study was considered comparable with that usually observed after SC injections of drugs other than anticoagulants. In a study of injections of placebo or erythropoietin, two hematomas at the injection site were recorded after 120 injections $(1.7 \%) .{ }^{22}$ In contrast, one study reported a $25 \%$ rate of hematoma after SC injection of low molecular weight heparin using a standard 26-gauge needle. ${ }^{18}$

Compliance with IFU for the self-injection system was evaluated at $93 \%$, which was slightly, but significantly, higher among younger than older subjects. Regardless, an overall rate of over $90 \%$ compliance was considered good, considering that the subjects were initially naïve at performing self-injections (excluding initial study training). Subject adaptability to using the self-injection system was considered a good predictor of ease of use. Moreover, the positive attitudes of the subjects towards self-injections also reflected their acceptance of the autoinjector. Attitudes reflecting fear of pain generally lead to poor treatment compliance and avoidance behavior, while compliance may be improved by confidence building and reassurance. ${ }^{23}$

All 40 subjects selected self-injection at the end of the study, suggesting that the autoinjector is the preferred injection system. Subjects also selected self-injections using the autoinjector when asked which combination of operator and system they would prefer for weekly SC injections to control 
a chronic disease. The autoinjector handled by a nurse was ranked second, indicating that the autoinjector was preferred over the syringe, regardless of who is performing the injection. Preference for the autoinjector is probably a result of the system's features, such as low perceived pain, practicability, and ease of use. The results require further investigation in patients with chronic diseases and with injectable drugs, and relative to other autoinjectors with different features.

\section{Conclusion}

In terms of technical performance, self-injection with the BD Physioject was not significantly different from injections using similar but "naked" syringes administered by nurses. There was no significant difference in the depth of the injection fluid depot between the two systems. Neither injection volume nor body site effected performance.

Self-injection with the BD Physioject was associated with greater acceptance, which was likely related to its selfinjector features, including a low perception of pain, regardless of the site of administration (abdomen or thigh). Body site, gender, and injection volume did not affect perceived pain or acceptance.

A few skin reactivity events (mainly some visible spots and drops of blood at the injection site), and a few adverse events of mild intensity which spontaneously resolved at the end of the study were observed, and demonstrated good clinical tolerance and good local skin tolerance of the two systems.

The observed errorless handling of the autoinjector when giving self-injections, in compliance with the IFU, suggests that the system is convenient and easy to use.

In conclusion, this study demonstrated that self-injection with the BD Physioject is an efficient, safe, and intuitive procedure, very well accepted by subjects and preferred to an injection by a nurse. Improved treatment compliance may therefore be anticipated in patients with chronic disease requiring injectable treatment, based on the subjects' positive attitudes and spontaneously expressed preference for the autoinjector.

\section{Acknowledgments}

The authors thank Dr Marie Steidel for performing and interpreting the ultrasound examinations, the clinical investigation staff of Eurofins Optimed for their help in conducting the clinical study and Nathalie Dalle (Clinical Investigation Center, Clermont-Ferrand University Hospital) for the build-up and analysis of psychologic acceptance.

\section{Disclosure}

The study was funded and sponsored by BD Medical Pharmaceutical Systems. BC, SF, AH, BJ, CJ, FC, and LP are employees of BD Medical Pharmaceutical Systems. DY and LM are employees of Eurofins Optimed.

\section{References}

1. Gabriel SE, Michaud K. Epidemiological studies in incidence, prevalence, mortality, and comorbidities of the rheumatic diseases. Arthritis Res Ther. 2009;11:229-244.

2. Lugaresi A. Addressing the need for increased adherence to multiple sclerosis therapy: Can delivery technology enhance patient motivation? Expert Opin Drug Deliv. 2009;6:995-1002.

3. diMatteo MR. Variations in patient's adherence to medical recommendation: A quantitative review of 50 years of research. Med Care. 2004;42:200-209.

4. Mohr DC, Cox D, Epstein L, Boudewyn A. Teaching patients to selfinject: Pilot study of a treatment for injection anxiety and phobia in multiple sclerosis patients prescribed injectable medication. J Behav Ther Exp Psychiatry. 2002;33:39-47.

5. Laurent PE, Pettis R, Easterbrook W, Berube J. Evaluating new hypodermic and intradermal injection devices. Med Device Technol. 2006; 17:75-78.

6. Collins SL, Moore RA, McQuay HJ. The visual analogue pain intensity scale: What is moderate pain in millimeters? Pain. 1997;72:95-97.

7. Jaber A, Driebergen R, Giovannoni G, Schellekens H, Simsarian J, Antonelli M. The Rebif ${ }^{\circledR}$ new formulation story. Drugs $R$ D. 2007;8: 335-348.

8. Likert R. The method of constructing an attitude scale. In: Fishbein M, editor. Readings in Attitude Theory and Measurement. New York, NY: Wiley; 1967.

9. Eagly A, Chaiken S. Attitude strength, attitude structure and resistance to change. In: Petty R, Kosnik J, editors. Attitude Strength. Mahwah, NJ: Erlbaum; 1995.

10. Shrigley RL, Koballa Jr TR. Attitude measurement: Judging the emotional intensity of likert-type science attitude statements. J Res Sci Teach. 1984;21:111-118.

11. Lebrun-Frenay C. [Neuroepidemiology, editorial]. Revue Neurol (Paris). 2009;165:613-614. French.

12. Calin A. The epidemiology of rheumatoid disease: Past and present. Dis Markers. 1986;4:1-6.

13. Cash CJ, Berman LH, Treece GM, Gee AH, Prager RW. Two- and threedimensional ultrasound in the development of a needle-free injection system. Br J Radiol. 2004;77:236-242.

14. Thow JC, Coulthard A, Home PD. Insulin injection site tissue depths and localization of a simulated insulin bolus using a novel air contrast ultrasonographic technique in insulin treated diabetic subjects. Diabet Med. 1992;9:915-920.

15. Sieg P, Hakim SG, Bierwolf S, Hermes D. Subcutaneous fat layer in different donor regions used for harvesting microvascular soft tissue flap in slender and adipose patients. Int J Oral Maxillofac Surg. 2003;32: 544-547.

16. Tarulli AW, Chin AB, Lee KS, Rutkove SB. Impact of skin-subcutaneous fat layer thickness on electrical impedance myography measurements: An initial assessment. Clin Neurophysiol. 2007;118: 2393-2397.

17. Main KM, Jørgensen JT, Hertel NT, Jensen S, Jakobsen L. Automatic needle insertion diminishes pain during growth hormone injection. Acta Paediatr. 1995;84:331-334.

18. Chan H. Effects of injection duration on site-pain intensity and bruising associated with subcutaneous heparin. J Adv Nurs. 2001;35: 882-892.

19. Laursen T, Hansen B, Fisker S. Pain perception after subcutaneous injections of media containing different buffers. Basic Clin Pharmacol Toxicol. 2006;98:218-221. 
20. Berthoux F, Ryckelynck JP, Rouanet S, et al; Painless Investigators Coordinators. A trial comparing local pain after subcutaneous injection of epoetin- $\beta$ versus darbepoetin- $\alpha$ in healthy volunteers. Clin Nephrol. 2008;70:33-40.

21. Pannier A, Jordan P, Dougherty FC, Bour F, Reignier B. Subcutaneous injection pain with C.E.R.A., a continuous erythropoietin receptor activator, compared with darbopoetin alfa. Curr Med Res Opin. 2007;23: 3025-3032.
22. Robb DM, Kanji Z. Comparison of two needle sizes for subcutaneous administration of enoxaparin: Effects on size of hematomas and pain on injection. Pharmacotherapy. 2002;22:1105-1109.

23. Consoli SM, Safar ME. Predictive value of patient psychological profile and type of physician-patient relationship in compliance of antihypertensive treatment. Arch Mal Coeur Vaiss. 1988;81S:145-150. French.

\section{Publish your work in this journal}

Patient Preference and Adherence is an international, peer-reviewed, open access journal focusing on the growing importance of patient preference and adherence throughout the therapeutic continuum. Patient satisfaction, acceptability, quality of life, compliance, persistence and their role in developing new therapeutic modalities and compounds to optimize clinical outcomes for existing disease states are major areas of interest. This journal has been accepted for indexing on PubMed Central. The manuscript management system is completely online and includes a very quick and fair peer-review system. Visit http://www.dovepress.com/ testimonials.php to read real quotes from published authors.

Submit your manuscript here: http://www.dovepress.com/patient-preference-and-adherence-journal 